\title{
Approximation Algorithms for Many-to-Many Traffic Grooming in WDM Mesh Networks
}

\author{
Mohammad A. Saleh Ahmed E. Kamal \\ Department of Electrical and Computer Engineering, Iowa State University, Ames, IA 50011 \\ Email: \{msaleh,kamal $\} @$ iastate.edu
}

\begin{abstract}
A large number of network applications today allow several users to interact together using the many-to-many service mode. In many-to-many communication, also referred to as group communication, a session consists of a group of users (we refer to them as members), where each member transmits its traffic to all other members in the same group. In this paper, we address the problem of grooming sub-wavelength many-to-many traffic (e.g., OC-3) into high-bandwidth wavelength channels (e.g., OC-192) in WDM mesh networks.

The cost of a WDM network is dominated by the cost of higher layer electronic ports (i.e., transceivers). A transceiver is needed for each initiation and termination of a lightpath. Therefore, our objective is to minimize the total number of lightpaths established. Unfortunately, the grooming problem even with unicast traffic has been shown to be NP-hard. For a number of special cases where the many-to-many traffic grooming problem is tractable, we efficiently derive the optimal solution, while in the general case, we introduce two novel approximation algorithms. We also consider the routing and wavelength assignment problem with the objective of minimizing the number of wavelengths used. Through extensive experiments, we show that the two algorithms use a number of lightpaths that is very close to that of a derived lower bound. Also, we compare the two algorithms on the several costs mentioned in the paper including the number of lightpaths and the number of wavelengths used.
\end{abstract}

\section{INTRODUCTION}

In wavelength routing networks, using wavelength division multiplexing (WDM), it is feasible to have hundreds of wavelengths per fiber each operating at 10 to $40 \mathrm{Gbps}$. Bandwidth requirements of user sessions, however, are usually of subwavelength granularities. For example, an MPEG compressed HDTV channel requires less than 20 Mbps of bandwidth. In order to reduce this huge bandwidth gap, traffic grooming was introduced to allow a number of sessions with sub-wavelength granularities to share the bandwidth of a wavelength channel.

Early network applications such as TELNET and FTP are characterized as unicast or "one-to-one". A large portion of network applications today, however, are of the multipoint type. For example, video distribution and file distribution are examples of multicast or "one-to-many" applications, while resource discovery and data collection are examples of manyto-one or "inverse multicasting" applications. Recently, another set of multipoint network applications has emerged such as multimedia conferencing, e-science applications, distance learning, distributed simulations, and collaborative processing [23]. In these applications, each of the participating entities

This research was supported in part by grant CNS-0626741 from the National Science Foundation.

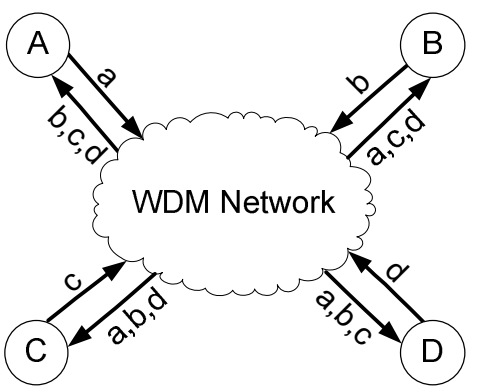

Fig. 1. A many-to-many session with members $\{A, B, C, D\}$ each with traffic denoted as $a, b, c$ and $d$, respectively.

both contributes and receives information to and from the other entities in the same communication session, and therefore are characterized as "many-to-many". In many-to-many communication, also referred to as group communication [22], a session consists of a group of users (we refer to them as members), where each member transmits its traffic to all other members in the same group (see Fig. 1).

Most of the early work on traffic grooming has focused on unicast traffic on ring and mesh topologies. Since a large portion of network applications today are of the multipoint type, many of the recent studies on traffic grooming has focused on multicast and many-to-one traffic types. In this work, we consider the many-to-many traffic grooming problem in WDM mesh networks, which is an important new research problem. In this problem, a collection of many-to-many session requests, each with an arbitrary sub-wavelength traffic demand, are given and the objective is to find a set of lightpaths and to find the corresponding routing and grooming of each of the traffic demands onto these lightpaths. For a complete design of the WDM network, the traffic grooming problem is followed by the routing and wavelength assignment (RWA) problem which finds routes and assign wavelengths to each of the lightpaths on the WDM network. The objective of the RWA problem is to minimize the total number of wavelengths used. The RWA has been extensively studied in the literature and it has been shown to be NP-complete. Therefore, in this work, we focus on the many-to-many traffic grooming problem. However, for completeness and for comparison purposes, we use one of the best existing heuristics for the RWA problem (the LFAP heuristic [18]).

The cost of a WDM network is dominated by the cost of higher layer electronic ports such as IP router ports, MPLS 
Label Switching Router (LSR) ports and SONET ADM ports (we refer to these ports as transceivers). A transceiver is needed for each initiation and termination of a lightpath (i.e, each lightpath requires two transceivers). Therefore, our objective in the many-to-many traffic grooming problem is to minimize the total number of lightpaths established.

\section{A. Problem Statement:}

An input to the many-to-many traffic grooming problem is a WDM network with an arbitrary physical topology represented by an undirected graph $G(V, E)$ with a set of nodes $V$ numbered $0,1, \ldots, N-1(N=|V|)$ and a set of links $E$. Each undirected link corresponds to two unidirectional fibers in opposite directions. The number of wavelengths per fiber is the same among all fibers and is denoted by $W_{\max }$, while the bandwidth capacity of a wavelength channel (i.e., the grooming factor) is $g$ units of traffic (the unit of traffic may be, e.g., an OC-3 circuit). Another input to the many-to-many traffic grooming problem is a collection of $K$ many-to-many session requests, where each session $s_{k}(1 \leq k \leq K)$ has a set of members $m_{s_{k}} \subseteq V$ with cardinality $N_{s_{k}}=\left|m_{s_{k}}\right|$. We assume uniformity of traffic within the same session, that is, each member in $m_{s_{k}}$ has the same traffic demand $t_{s_{k}}$, where $1 \leq t_{s_{k}} \leq g$. Let $N_{\text {min }}$ and $t_{\text {min }}$ denote the minimum session size and the minimum traffic demand among all the $K$ sessions, respectively and let $k_{i}$ denote the number of sessions where node $i$ is a member.

The output of the many-to-many traffic grooming problem is a set of lightpaths and the corresponding routing and grooming of each of the many-to-many traffic demands onto these lightpaths. More precisely, the output is the number of lightpaths $P_{i j}$ to be established between each ordered pair of nodes $(i, j)$, where $i, j \in V$, and the sequence of lightpaths that each traffic stream follows. Note that for each member in a many-to-many session $s_{k}$ there is a traffic stream of $t_{s_{k}}$ traffic units that needs to be delivered to all other members in the same session.

The objective of the many-to-many traffic grooming problem is to minimize the total number of lightpaths established $P\left(P=\sum_{i, j} P_{i j}\right)$. It was shown in [7] that the unicast traffic grooming problem without the RWA problem is NPhard. Therefore, the many-to-many traffic grooming problem is NP-hard since it is at least as hard as the unicast one.

\section{B. Related Work:}

Traffic grooming has been extensively studied for unicast traffic [1]-[10]. Some of the studies were restricted to ring topologies [6], [3], [5], while others were for general mesh topologies [1], [2], [8], [9], [10], [4]. In [6], the authors addressed the traffic grooming problem on a number of WDM ring architectures with the objective of minimizing the overall network cost. In [5], the authors proposed optimal and nearoptimal algorithms for traffic grooming in SONET WDM rings with the objective of minimizing the number of wavelengths and SONET ADMs. In [4], the authors proposed an auxiliary graph model for traffic grooming in heterogeneous WDM mesh networks and developed an integrated traffic grooming algorithm that jointly solves the traffic grooming subproblems. In [1], the authors showed that the traffic grooming problem is APX-hard, which means that the optimum cannot be approximated arbitrarily closely. They also proposed approximation algorithms for minimizing the total equipment cost and for minimizing the lightpath count. In [2], the authors provided a hierarchical framework for traffic grooming in a WDM mesh network. For a survey of advances in unicast traffic grooming, the reader is referred to [10].

Traffic grooming has also been considered for multicast traffic [11]-[16]. Similar to unicast traffic, some of the studies were restricted to ring topologies [15], [16], while others were for general mesh topologies [11], [12], [14], [13]. In [15], the authors addressed the multicast traffic grooming problem in metropolitan WDM ring networks with the objective of minimizing the electronic copying. In [16], the authors introduced a graph based heuristic for the multicast traffic grooming problem in unidirectional SONET/WDM rings and compared it to the multicast extension of the best known unicast traffic grooming heuristic in [5]. In [11] and [20], the authors addressed the multicast and the many-to-one traffic grooming problems, respectively in WDM mesh networks. They provided MILP formulations and also developed heuristic solutions. For a survey of advances in multicast traffic grooming, the reader is referred to [13].

To the best of our knowledge, many-to-many traffic grooming is a new research problem that has been only considered in [17], [21], [19]. In [21], the authors addressed the many-tomany traffic grooming problem in WDM ring networks with the objective of reducing the overall network cost. In our previous works [17], [19], MILP formulations and heuristic solutions were introduced for the many-to-many traffic grooming problem in WDM mesh networks. Although the MILPs guaranteed an optimal solution, their complexity was too high. Also, the heuristics introduced didn't guarantee any upper bound on the number of lightpaths required. To the best of our knowledge, this is the first work that proposes approximation algorithms for the many-to-many traffic grooming problem.

\section{Contributions and Paper Organization:}

In this paper, we study the many-to-many traffic grooming problem in WDM mesh networks, which is an important new research problem. Our contributions can be summarized as follows:

- In Section II, we introduce lightpath cycles (a formal definition will be given later) as the optimal solution for a number of special cases where the many-to-many traffic grooming problem is tractable.

- In Section III, we introduce two novel approximation algorithms for the general many-to-many traffic grooming problem. Algorithm 1, which is based on lightpath cycles, has an approximation ratio of $\min \{g, 1+$ $\left.\frac{g}{\left(N_{\min }-1\right) t_{\min }}, N-N_{\min }+1\right\}$. Algorithm 2, which is based on a hub node that collects and distributes traffic, has a 2-approximation ratio. We also consider the RWA 
TABLE I

LIST OF SYMBOLS USED IN THE PAPER

\begin{tabular}{|c|c|}
\hline Symbol & Definition \\
\hline$G(V, E)$ & $\begin{array}{l}\text { undirected graph with a set of nodes } V \text { and a set of links } E \text {, which } \\
\text { represents the physical topology of the WDM network. }\end{array}$ \\
\hline$N$ & number of nodes in the network $(N=|V|)$. \\
\hline$K$ & number of many-to-many sessions. \\
\hline$g$ & grooming factor. \\
\hline$W_{\max }$ & total number of wavelengths per fiber link. \\
\hline & total number of wavelengths used $\left(W \leq W_{\max }\right)$. \\
\hline$s_{k}$ & many-to-many session number $k(1 \leq k \leq K)$ \\
\hline$m_{s_{k}}$ & set of members in session $s_{k}$. \\
\hline$N_{s_{k}}$ & number of members in session $s_{k}\left(N_{s_{k}}=\left|m_{s_{k}}\right|\right)$. \\
\hline$t_{s_{k}}$ & traffic demand of members in session $s_{k}$. \\
\hline$t^{n}$ & the uniform traffic demand. \\
\hline$k_{i}$ & number of sessions where node $i$ is a member. \\
\hline$N_{\min }$ & minimum session size among all the $K$ sessions. \\
\hline$t_{\min }$ & minimum traffic demand among all the $K$ sessions. \\
\hline & set of sessions where node $i$ is a member $\left(k_{i}=\left|S_{i}\right|\right)$. \\
\hline$S_{i j}$ & set of sessions where both nodes $i$ and $j$ are members. \\
\hline$S_{i F j}$ & $\begin{array}{l}\text { set of sessions where member } i \text { follows member } j \text { immediately in the } \\
\text { session's LCs. }\end{array}$ \\
\hline$P_{i j}$ & total number of lightpaths from node $i$ to node $j$. \\
\hline$P$ & total number of lightpaths in the network $\left(P=\sum_{i, j} P_{i j}\right)$. \\
\hline rem $_{i j}$ & $\begin{array}{l}\text { remaining unused capacity on lightpaths from } i \text { to } j \text { if we place } j \\
\text { after } i \text { in the LCs for all sessions in the set } S_{i j} \text {. }\end{array}$ \\
\hline
\end{tabular}

problem with the objective of minimizing the total number of wavelengths used $W$.

- In Section IV, we conduct extensive experiments to show that Algorithms 1 and 2 use a number of lightpaths that is significantly close to that of a derived lower bound. We also compare Algorithms 1 and 2 on the several costs mentioned in the paper including the number of lightpaths and the number of wavelengths used.

- In Section V, we conclude the paper. For a quick reference, Table I lists all the symbols used in the paper.

\section{LightPATH CYCLES}

In this section, we efficiently derive the optimal solution for a number of special cases where the many-to-many traffic grooming problem is tractable. First, we have the following definition.

Definition 1. A lightpath cycle (LC) for a many-to-many session $s_{k}$ is a simple cycle of $N_{s_{k}}$ lightpaths that visits each member in $m_{s_{k}}$ exactly once.

An example of a LC for a many-to-many session $s_{k}$ with a set of members $m_{s_{k}}=\{A, B, C, D\}$ is shown in Fig. 2.(a). Note that LCs only describe a virtual topology and their mapping to the physical topology is part of the RWA problem. For a single many-to-many session $s_{k}$, LCs for $s_{k}$ serve as an optimal solution, as indicated by the following theorem:

Theorem 1. An optimal solution that minimizes the total number of lightpaths required to provision a single many-tomany session $s_{k}$ consists of $\left\lceil\frac{\left(N_{s_{k}}-1\right) t_{s_{k}}}{g}\right\rceil$ identically ordered LCs for $s_{k}$.

Proof: Any feasible solution to provision $s_{k}$ must have at least a total of $N_{s_{k}}\left\lceil\frac{\left(N_{s_{k}}-1\right) t_{s_{k}}}{g}\right\rceil$ lightpaths. This is due to the fact that each member in $m_{s_{k}}$ must have at least $\left\lceil\frac{\left(N_{s_{k}}-1\right) t_{s_{k}}}{g}\right\rceil$ lightpaths incoming to receive its traffic. Note

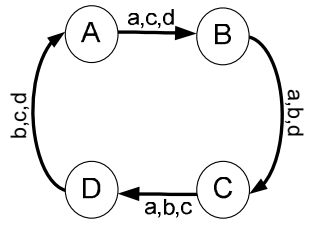

(a)

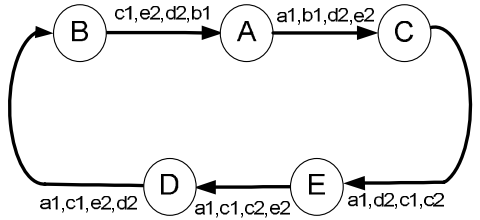

(b)
Fig. 2. (a): LC for a many-to-many session $s_{k}$ with a set of members $m_{s_{k}}=\{A, B, C, D\}$ each with one traffic unit denoted as $a, b, c$ and $d$, respectively $(g=3)$. (b): LC for many-to-many sessions $s_{1}$ and $s_{2}$ where $m_{s_{1}}=\{A, B, C\}$ each with one traffic unit denoted as $a 1, b 1, c 1$ and $m_{s_{2}}=\{C, D, E\}$ each with one traffic unit denoted as $c 2, d 2, e 2(g=4)$.

that $\left\lceil\frac{\left(N_{s_{k}}-1\right) t_{s_{k}}}{g}\right\rceil$ LCs for $s_{k}$ have exactly $N_{s_{k}}\left\lceil\frac{\left(N_{s_{k}}-1\right) t_{s_{k}}}{g}\right\rceil$ lightpaths. Therefore, if we prove it is a feasible solution then it will also be an optimal one. Now, by letting each member in $m_{s_{k}}$ transmit its traffic through the $\left[\frac{\left(N_{s_{k}}-1\right) t_{s_{k}}}{g}\right]$ identically ordered LCs for $s_{k}$ until it reaches the member just before it in the LCs (see Figure 2.(a)), we guarantee two things. First, exactly $\left(N_{s_{k}}-1\right) t_{s_{k}}$ traffic units are groomed between each pair of consecutive members in the LCs and therefore $\left\lceil\frac{\left(N_{s_{k}}-1\right) t_{s_{k}}}{g}\right\rceil$ lightpaths are sufficient to groom this traffic. Second, each member in $m_{s_{k}}$ receives the traffic from the other $N_{s_{k}}-1$ members in the same session. Therefore, $\left[\frac{\left(N_{s_{k}}-1\right) t_{s_{k}}}{g}\right]$ identically ordered LCs for $s_{k}$ is a feasible and an optimal solution.

Hence, for a single many-to-many session $s_{k}$, the total number of lightpaths required is:

$$
P=N_{s_{k}}\left\lceil\frac{\left(N_{s_{k}}-1\right) t_{s_{k}}}{g}\right\rceil
$$

Next, we consider two special cases of multiple many-to-many sessions where the many-to-many traffic grooming problem is tractable. The first special case, which follows directly from Theorem 1, is when the member sets of the many-tomany sessions are pairwise disjoint. In this case, we have the following theorem:

Theorem 2. An optimal solution that minimizes the total number of lightpaths required to provision a set of many-tomany sessions $s_{1}, s_{2}, \ldots ., s_{K}$ when $m_{s_{k}} \cap m_{s_{L}}=\phi$ for all $1 \leq k \leq K$ and $1 \leq l \leq K(k \neq l)$ consists of $\left\lceil\frac{\left(N_{s_{m}}-1\right) t_{s_{m}}}{g}\right\rceil$ identically ordered LCs for $s_{m}$, for all $1 \leq m \leq K$.

Proof: Since the member sets of the sessions are pairwise disjoint, then the argument made in the proof of theorem 1 can now be made to each of the sessions independently.

Hence, for this special case of multiple many-to-many sessions, the total number of lightpaths required is:

$$
P=\sum_{k=1}^{K} N_{s_{k}}\left\lceil\frac{\left(N_{s_{k}}-1\right) t_{s_{k}}}{g}\right\rceil
$$

The second special case of multiple many-to-many sessions where the many-to-many traffic grooming problem is tractable 
is when $\left[\frac{\sum_{k=1}^{K}\left(N_{s_{k}}-1\right) t_{s_{k}}}{g}\right\rceil=1$. First, we have the following definition.

Definition 2. A lightpath cycle (LC) for a set of many-tomany sessions $s_{1}, s_{2}, \ldots, s_{K}$ is a simple cycle of $\left|\bigcup_{k=1}^{K} m_{s_{k}}\right|$ lightpaths that visits each member in the union set $\bigcup_{k=1}^{\bar{K}} m_{s_{k}}$ exactly once.

An example of a LC for sessions $s_{1}$ and $s_{2}$ each with a set of members $m_{s_{1}}=\{A, B, C\}$ and $m_{s_{2}}=\{C, D, E\}$ is shown in Fig. 2.(b). A LC for a set of many-to-many sessions serves as an optimal solution in a special case, as indicated by the following theorem:

Theorem 3. An optimal solution that minimizes the total number of lightpaths required to provision a set of many-tomany sessions $s_{1}, s_{2}, \ldots, s_{K}$ when $\left[\frac{\sum_{k=1}^{K}\left(N_{s_{k}}-1\right) t_{s_{k}}}{g}\right]=1$ consists of a $L C$ for $s_{1}, s_{2}, \ldots, s_{K}$.

Proof: Any feasible solution to provision the set of sessions $s_{1}, s_{2}, \ldots, s_{K}$ must have at least a total of $\left|\bigcup_{k=1}^{K} m_{s_{k}}\right|$ lightpaths. This is due to the fact that each member in $\bigcup_{k=1}^{K} m_{s_{k}}$ must have at least one lightpath incoming to receive its traffic. Note that a LC for $s_{1}, s_{2}, \ldots, s_{K}$ has exactly $\left|\bigcup_{k=1}^{K} m_{s_{k}}\right|$ lightpaths. Therefore, if we prove it is a feasible solution then it will also be an optimal one. Now, by letting each member in $\bigcup_{k=1}^{K} m_{s_{k}}$ transmit its traffic through the LC until it reaches the last member interested in receiving this traffic (see Figure 2.(b)), we guarantee two things. First, exactly $\sum_{k=1}^{K}\left(N_{s_{k}}-1\right) t_{s_{k}}$ traffic units are groomed between each pair of consecutive members in the LC and since $\left\lceil\frac{\sum_{k=1}^{K}\left(N_{s_{k}}-1\right) t_{s_{k}}}{g}\right\rceil=1$, then a single lightpath is sufficient to groom this traffic. Second, each member in $\bigcup_{k=1}^{K} m_{s_{k}}$ receives the traffic from all the other $N_{s_{k}}-1$ members in all sessions $s_{k}$ where this member appears. Therefore, a LC for $s_{1}, s_{2}, \ldots, s_{K}$ is a feasible and an optimal solution.

Hence, for this special case of multiple many-to-many sessions, the total number of lightpaths required is:

$$
P=\left|\bigcup_{k=1}^{K} m_{s_{k}}\right|
$$

\section{Approximation Algorithms}

As we stated before, the general many-to-many traffic grooming problem is NP-hard. In this section, we introduce two novel approximation algorithms for the many-to-many traffic grooming problem in WDM mesh networks. First, we derive a lower bound on the number of lightpaths required.

\section{A. A lower bound}

We derive a lower bound on the number of lightpaths required by considering each node in the network separately. The minimum number of lightpaths incoming to a node $i$ can be found by counting the total traffic that this node should receive from all sessions $s_{k}$ where $i \in m_{s_{k}}$. Let $S_{i}$ denotes the set of sessions where node $i$ is a member (note that $\left|S_{i}\right|=k_{i}$ ). The total traffic that node $i$ should receive is $\sum_{s_{k} \in S_{i}}\left(N_{s_{k}}-1\right) t_{s_{k}}$.
Therefore, at least $\left\lceil\frac{\sum_{s_{k} \in S_{i}}\left(N_{s_{k}}-1\right) t_{s_{k}}}{g}\right\rceil$ lightpaths should be incoming to $i$ in order to receive this traffic. Summing for all the nodes in the network, we obtain a lower bound $L$ on the total number of lightpaths required:

$$
L=\sum_{i=0}^{N-1}\left\lceil\frac{\sum_{s_{k} \in S_{i}}\left(N_{s_{k}}-1\right) t_{s_{k}}}{g}\right\rceil
$$

We note that this is just a lower bound on the number of lightpaths and it does not necessarily yield a feasible solution to the many-to-many traffic grooming problem. Next, we obtain an upper bound on the number of lightpaths required by any many-to-many traffic grooming algorithm.

\section{B. An upper bound}

We consider the worst case scenario where no traffic grooming is performed between any two traffic streams even within the same session. In this case, each node $i$ will have a direct lightpath incoming from each of the other $N_{s_{k}}-1$ members in the same session $s_{k}$ for all sessions $s_{k} \in S_{i}$. Therefore, the total number of lightpaths $P$ required according to this worst case scenario is given by:

$$
\begin{aligned}
P= & \sum_{i=0}^{N-1} \sum_{s_{k} \in S_{i}} N_{s_{k}}-1 \leq \sum_{i=0}^{N-1} \sum_{s_{k} \in S_{i}}\left(N_{s_{k}}-1\right) t_{s_{k}} \\
& \leq \sum_{i=0}^{N-1} g\left\lceil\frac{\sum_{s_{k} \in S_{i}}\left(N_{s_{k}}-1\right) t_{s_{k}}}{g}\right\rceil=g L
\end{aligned}
$$

Since this is the worst case scenario, then it serves as an upper bound for any many-to-many traffic grooming algorithm. Hence, we have the following result:

Theorem 4. Any many-to-many traffic grooming algorithm with any grooming policy is a g-approximation algorithm.

Next, we propose two novel approximation algorithms for the many-to-many traffic grooming problem in WDM mesh networks.

\section{Algorithm 1}

In this algorithm, we assume that many-to-many sessions are provisioned through lightpath cycles (LCs). Although the optimality of LCs was only for certain special cases, we will show that this assumption generally gives near-optimal solutions. First, let us assume that each session $s_{k}$ is provisioned through $\left[\frac{\left(N_{s_{k}}-1\right) t_{s_{k}}}{g}\right]$ identically ordered LCs for $s_{k}$ and ignore inter-session grooming (LCs in this case only perform intra-session grooming between members within the same session, see Fig. 2.(a)). In this case, node $i$ will have $\left\lceil\frac{\left(N_{s_{k}}-1\right) t_{s_{k}}}{g}\right\rceil$ lightpaths incoming from each session $s_{k} \in S_{i}$. Hence, the total number of lightpaths $P$ required according to this algorithm is given by:

$$
P=\sum_{i=0}^{N-1} \sum_{s_{k} \in S_{i}}\left\lceil\frac{\left(N_{s_{k}}-1\right) t_{s_{k}}}{g}\right\rceil
$$




$$
\begin{gathered}
\leq \sum_{i=0}^{N-1}\left(\left\lceil\frac{\sum_{s_{k} \in S_{i}}\left(N_{s_{k}}-1\right) t_{s_{k}}}{g}\right\rceil+k_{i}\right) \\
=L+\sum_{i=0}^{N-1} k_{i}
\end{gathered}
$$

The inequality holds due to the fact that $\sum_{m=0}^{M-1}\left\lceil x_{m}\right\rceil \leq$ $\left\lceil\sum_{m=0}^{M-1} x_{m}\right\rceil+M$ for any positive integer $M$ and positive real values $x_{1}, x_{2}, \ldots, x_{M-1}$.

Now, lets consider the lower bound $L$ again:

$$
\begin{aligned}
L & =\sum_{i=0}^{N-1}\left\lceil\frac{\sum_{s_{k} \in S_{i}}\left(N_{s_{k}}-1\right) t_{s_{k}}}{g}\right\rceil \\
\geq & \sum_{i=0}^{N-1}\left\lceil\frac{\sum_{s_{k} \in S_{i}}\left(N_{m i n}-1\right) t_{m i n}}{g}\right\rceil \\
\geq & \sum_{i=0}^{N-1} \frac{\sum_{s_{k} \in S_{i}}\left(N_{\text {min }}-1\right) t_{\text {min }}}{g} \\
& =\frac{\left(N_{\text {min }}-1\right) t_{\text {min }}}{g} \sum_{i=0}^{N-1} k_{i}
\end{aligned}
$$

Substituting (4) in (3), we have:

$$
\begin{aligned}
P \leq L & +\sum_{i=0}^{N-1} k_{i} \leq L+\frac{L g}{\left(N_{\min }-1\right) t_{\min }} \\
& =\left(1+\frac{g}{\left(N_{\min }-1\right) t_{\min }}\right) L
\end{aligned}
$$

Hence, we have the following result:

Theorem 5. Any many-to-many traffic grooming algorithm that assumes that each session $s_{k}$ is provisioned through $\left\lceil\frac{\left(N_{s_{k}}-1\right) t_{s_{k}}}{g}\right\rceil$ identically ordered LCs for $s_{k}$ is a $1+$ $\frac{g}{\left(N_{\min }-1\right) t_{\min }}$ approximation algorithm.

An interesting case is when $\left(N_{\min }-1\right) t_{\min } \geq g$ where we obtain an approximation ratio of at most 2 . This relatively good approximation ratio is intuitive since when $\left(N_{\min }-1\right) t_{\min } \geq$ $g$, then each session's traffic efficiently fills at least half of its LCs. The best approximation ratio we can obtain is when $N_{\text {min }}=N$ and $t_{\min }=g$ where we get an approximation ratio of $\left(1+\frac{1}{(N-1)}\right)$. On the other extreme, when $\left(N_{\min }-\right.$ 1) $t_{\min }$ is too small (e.g., equals to 1$)$, then we obtain a $1+g$ approximation ratio. This is also intuitive since when $\left(N_{\min }-\right.$ 1) $t_{\text {min }}=1$, then $\left[\frac{\left(N_{s_{k}}-1\right) t_{s_{k}}}{g}\right]$ LCs for each session $s_{k}$ may be a significant waste without inter-session grooming.

To further improve this algorithm we still assume that each session $s_{k}$ is provisioned through $\left[\frac{\left(N_{s_{k}}-1\right) t_{s_{k}}}{g}\right]$ identically ordered LCs for $s_{k}$. However, we now perform inter-session grooming so that LCs of different sessions may share lightpaths (i.e., lightpaths may groom traffic from different sessions and not just traffic from differnet members within the same session). The algorithm performs inter-session grooming as follows. Between each pair of nodes $i$ and $j$, it grooms the $\sum_{s_{k}}\left(N_{s_{k}}-1\right) t_{s_{k}}$ traffic units for all sessions $s_{k}$ where $i, j \in m_{s_{k}}$ and member $j$ follows member $i$ immediately in the session's LCs. Note that the order of the members in the LCs is significant and must be taken into account to make inter-session grooming efficient. We start by assuming that members are ordered randomly in each session's LCs. Let $S_{i F j}$ denotes the set of sessions where member $i$ follows member $j$ immediately in the session's LCs. The number of lightpaths $P$ required according to this algorithm is given by:

$$
\begin{gathered}
P=\sum_{i=0}^{N-1} \sum_{j=0 ; j \neq i}^{N-1}\left\lceil\frac{\sum_{s_{k} \in S_{i F j}}\left(N_{s_{k}}-1\right) t_{s_{k}}}{g}\right\rceil \\
\leq \sum_{i=0}^{N-1} \sum_{j=0 ; j \neq i}^{N-1}\left\lceil\frac{\sum_{s_{k} \in S_{i}}\left(N_{s_{k}}-1\right) t_{s_{k}}}{g}\right\rceil \\
=\sum_{j=0}^{N-2} \sum_{i=0}^{N-1}\left\lceil\frac{\sum_{s_{k} \in S_{i}}\left(N_{s_{k}}-1\right) t_{s_{k}}}{g}\right\rceil \\
=(N-1) L
\end{gathered}
$$

The first inequality holds since $S_{i F j}$ is a subset of $S_{i}$. The exchange of the summations in the second equality is valid since what is inside the inner summation $\left\lceil\frac{\sum_{s_{k} \in S_{i}}\left(N_{s_{k}}-1\right) t_{s_{k}}}{g}\right\rceil$ is independent of $j$. This algorithm so far has an approximation ratio of $\min \left\{g, 1+\frac{g}{\left(N_{\min }-1\right) t_{\text {min }}}, N-1\right\}$. A better approximation ratio can be found by making a more intelligent ordering of the members in each session's LCs. We first order the nodes in the network in a list according to some criteria (e.g., ascending or descending order). Afterwards, for each session $s_{k}$, we order members in the session's LCs according to the list of ordered nodes. More precisely, we order members in a session $s_{k}$ LCs by placing the first member as the first node in the list that is a member in session $s_{k}$ and the second member as the second node in the list that is a member in session $s_{k}$ and so on until we place all the members. Note that the first member immediately follows the last member in the ordered LCs. Based on this ordering of the members in the sessions' LCs, we have the following lemma:

Lemma 6. A node $i$ in Algorithm 1 cannot have direct lightpaths incoming from more than $N-N_{\min }+1$ other nodes.

Proof: We prove the lemma by proving that the $N_{\min }-2$ nodes that immediately follow $i$ in the list of ordered nodes cannot have direct lightpaths outgoing to $i$ (note that the first node in the list immediately follows the last node in the list). To prove this, we consider any node $j$ in these $N_{\min }-2$ nodes. We have two cases for $j$. Either $j$ comes after $i$ in the list (i.e., between $i$ and the last node in the list) or before $i$ in the list (i.e., between the first node in the list and $i$ ).

In the first case when $j$ comes after $i$ in the list, the only way that $j$ could have a direct lightpath outgoing to $i$ is when $i$ is the first member in the LCs for a session and $j$ is the last one. Since the session size is at least $N_{\min }$ then there should be at least $N_{\min }-2$ other nodes in the session. Also, since members in the LCs are ordered according to the list of 
ordered nodes, then these $N_{\min }-2$ nodes must be between $i$ and $j$ in the list. However, there are at most $N_{\min }-3$ nodes between $i$ and $j$ which makes a contradiction.

In the second case when $j$ comes before $i$ in the list, the only way that $j$ could have a direct lightpath outgoing to $i$ is when $j$ immediately precedes $i$ in the LCs for a session. This prevents all the nodes between $j$ and $i$ in the list (which are at least $\left.N-\left(N_{\min }-2\right)-1=N-N_{\min }+1\right)$ to be members in the session. Hence, only $N-\left(N-N_{\min }+1\right)-$ $2=N_{\min }-3$ nodes are left to be members in the session. However, since the session size is at least $N_{\min }$ then there should be at least $N_{\min }-2$ other nodes in the session, which makes a contradiction. Therefore, the $N_{\min }-2$ nodes that immediately follow $i$ in the list of ordered nodes cannot have direct lightpaths outgoing to $i$, which means that $i$ cannot have direct lightpaths incoming from more than $N-N_{\min }+1$ other nodes.

After this ordering of the members in each session's LCs, between each pair of nodes $i$ and $j$, Algorithm 1 grooms the $\sum_{s_{k}}\left(N_{s_{k}}-1\right) t_{s_{k}}$ traffic units for all sessions $s_{k} \in S_{j F i}$. The number of lightpaths $P$ required by Algorithm 1 is given by:

$$
P=\sum_{i=0}^{N-1} \sum_{j=0 ; j \neq i}^{N-1}\left\lceil\frac{\sum_{s_{k} \in S_{i F j}}\left(N_{s_{k}}-1\right) t_{s_{k}}}{g}\right\rceil
$$

However, from lemma $6, j$ cannot take more than $N-N_{\min }+1$ values and since $S_{i F j}$ is a subset of $S_{i}$, then we have:

$$
\begin{gathered}
P \leq \sum_{i=0}^{N-1} \sum_{j=0}^{N-N_{\min }}\left\lceil\frac{\sum_{s_{k} \in S_{i}}\left(N_{s_{k}}-1\right) t_{s_{k}}}{g}\right\rceil \\
=\sum_{j=0}^{N-N_{\min }} \sum_{i=0}^{N-1}\left\lceil\frac{\sum_{s_{k} \in S_{i}}\left(N_{s_{k}}-1\right) t_{s_{k}}}{g}\right\rceil \\
=\left(N-N_{\text {min }}+1\right) L
\end{gathered}
$$

Therefore, we have the following result:

Theorem 7. Algorithm 1 is a $\min \left\{g, 1+\frac{g}{\left(N_{\text {min }}-1\right) t_{\min }}, N-\right.$ $\left.N_{\text {min }}+1\right\}$ approximation algorithm.

Note that when $N_{\min }=N$ (i.e., all-to-all communication), then Algorithm 1 guarantees an optimal solution. On the other extreme when $N_{\text {min }}=2$, then we are back to the $\min \{g, 1+$ $\left.\frac{g}{\left(N_{\min }-1\right) t_{\min }}, N-1\right\}$ approximation ratio.

Although any order of the nodes in the network will guarantee the above approximation ratio, Algorithm 1 orders the nodes in a way to make inter-session grooming efficient (the full description of the algorithm is shown in Algorithm 1). For each ordered pair of nodes $(i, j)$, the algorithm computes the $\mathrm{rem}_{i j}$ value (lines 3-8) which represents the remaining unused capacity on lightpaths from $i$ to $j$ if we place $j$ after $i$ in the LCs for all sessions in the set $S_{i j}$. If this value is low (e.g., close to 0 ), then placing $j$ after $i$ results in an efficient grooming of traffic into lightpaths. However, when this value is high (e.g., close to $g-1$ ), then placing $j$ after $i$ results in an inefficient grooming where lightpaths are low utilized.

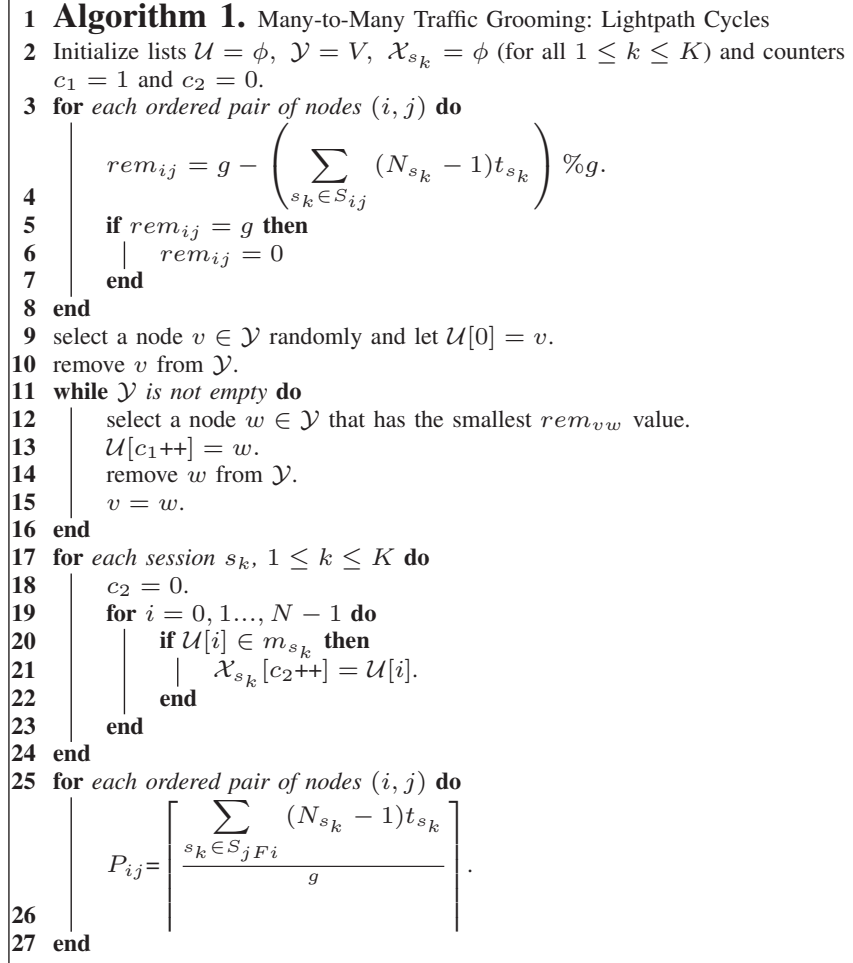

The algorithm then orders the nodes in the network in the list $\mathcal{U}$ (lines 9-16) according to the $\mathrm{rem}_{i j}$ values as follows. It selects the first node $v$ in the list randomly and then places the next node $w$ in the list as the node with the smallest rem $_{v w}$ value and it keeps doing this until it selects all the nodes in the network. Afterwards, for each session $s_{k}$, the algorithm orders members in the session's LCs in the list $\mathcal{X}_{s_{k}}$ (lines 17-24) as follows. It places the first member in $\mathcal{X}_{s_{k}}$ as the first node in the list $\mathcal{U}$ that is a member in session $s_{k}$ and the second member in $\mathcal{X}_{s_{k}}$ as the second node in the list $\mathcal{U}$ that is a member in session $s_{k}$ and it keeps doing this until it places all the members. Finally, the algorithm computes the total number of lightpaths needed between each ordered pair of nodes $(i, j)$ to groom the total traffic $\sum_{s_{k}}\left(N_{s_{k}}-1\right) t_{s_{k}}$ from all sessions $s_{k} \in S_{j F i}$ (lines 25-27).

\section{Algorithm 2}

In this algorithm, a hub node $h$ is chosen from the set of nodes in the network. The traffic between any two members in a many-to-many session is routed as follows. First, the traffic is routed through a direct lightpath from the first member to the hub and then through a direct lightpath from the hub to the second member. Note that when the hub is the first member then the first step is not needed and when it is the second member then the second step is not needed. According to this algorithm, for each node $i \neq h$ to receive all its traffic, it needs $\left\lceil\sum_{s_{k} \in S_{i}} \frac{\left(N_{s_{k}}-1\right) t_{s_{k}}}{g}\right\rceil$ lightpaths incoming from the hub and it needs $\left\lceil\sum_{s_{k} \in S_{i}} \frac{t_{s_{k}}}{g}\right\rceil$ lightpaths outgoing to the hub to send all its traffic. Therefore, the total number of lightpaths $P$ 


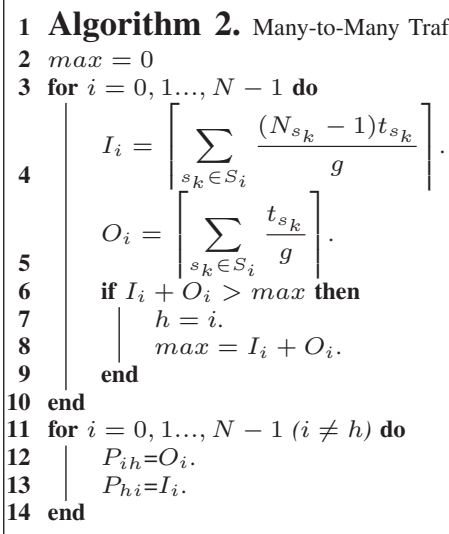

required according to this algorithm is given by:

$$
\begin{gathered}
P=\sum_{i=0 ; i \neq h}^{N-1}\left(\left\lceil\sum_{s_{k} \in S_{i}} \frac{\left(N_{s_{k}}-1\right) t_{s_{k}}}{g}\right\rceil+\left\lceil\sum_{s_{k} \in S_{i}} \frac{t_{s_{k}}}{g}\right\rceil\right) \\
\leq \sum_{i=0 ; i \neq h}^{N-1}\left\lceil\sum_{s_{k} \in S_{i}} \frac{\left(N_{s_{k}}-1\right) t_{s_{k}}}{g}\right\rceil+\sum_{i=0 ; i \neq h}^{N-1}\left\lceil\sum_{s_{k} \in S_{i}} \frac{\left(N_{s_{k}}-1\right) t_{s_{k}}}{g}\right\rceil \\
=2 L
\end{gathered}
$$

Therefore, we have the following result:

Theorem 8. Algorithm 2 is a 2-approximation algorithm.

Note that the optimal way to select the hub node is to select the node $h$ with the largest $\left\lceil\sum_{s_{k} \in S_{h}} \frac{\left(N_{s_{k}}-1\right) t_{s_{k}}}{g}\right\rceil+$ $\left\lceil\sum_{s_{k} \in S_{h}} \frac{t_{s_{k}}}{g}\right\rceil$ value. This minimizes the total number of lightpaths in the network. The full description of this algorithm is shown in Algorithm 2. The algorithm first computes the values of $I_{i}$ and $O_{i}$ for all the nodes in the network and selects the hub node $h$ as the node with the largest $I_{i}+O_{i}$ value (lines 3-10). Afterwards, the algorithm computes the total number of lightpaths needed between each node and the hub and between the hub and each node (lines 11-14).

\section{E. Complexity Analysis}

Algorithm 1 requires a preprocessing step that constructs the sets $S_{i j}$. This step requires visiting all the $K$ sessions for each pair $(i, j)$, which in total requires $O\left(K N^{2}\right)$ time. Once these sets are constructed, then the $r e m_{i j}$ values can be computed in $O\left(N^{2}\right)$ time (lines 3-8). Afterwards, the list $\mathcal{U}$ is constructed in $O\left(N^{2}\right)$ time (lines 9-16) and the lists $\mathcal{X}_{s_{k}}$ are constructed in $O(K N)$ time (lines 17-24). Then, Algorithm 1 needs to construct the the sets $S_{j F i}$. This requires visiting all the members in all the lists $\mathcal{X}_{s_{k}}$ for each pair $(i, j)$, which in total requires $O\left(K N^{3}\right)$ time. Once these sets are constructed, then the $P_{i j}$ values can be computed in $O\left(N^{2}\right)$ time (lines 25-27). This drives the time complexity of Algorithm 1 to $O\left(K N^{3}\right)$. Algorithm 2, on the other hand, requires a preprocessing step that constructs the sets $S_{i}$. This step requires visiting all the $K$ sessions for each node $i$, which in total requires $O(K N)$ time. Once these sets are constructed, then the $I_{i}, O_{i}$ and $h$ values

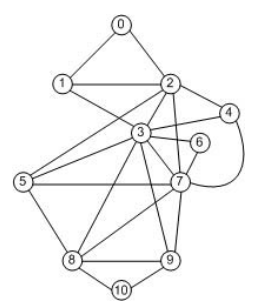

(a) NJ-LATA

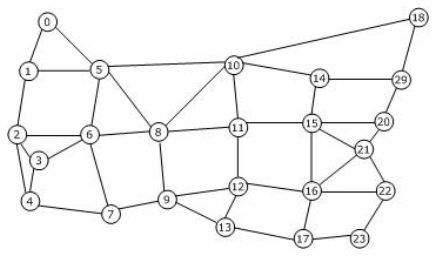

(b) USNET
Fig. 3. Networks used in the results

can be computed in $O(N)$ time (lines 3-10). Afterwards, the $P_{i h}$ and $P_{h i}$ values are computed in $O(N)$ time (lines 11-14). This drives the time complexity of Algorithm 2 to $O(K N)$.

\section{F. Routing and Wavelength Assignment}

Once we solve the many-to-many traffic grooming problem and determine the set of lightpaths to be established, we can then consider the routing and wavelength assignment (RWA) problem. In this problem, we need to provision each of the lightpaths on the WDM network by determining: 1) the physical route of each lightpath on the network, and 2) the wavelength to assign to each lightpath while taking the wavelength continuity constraint into account. The objective is to minimize the total number of wavelengths used $W$.

It is to be noted that the RWA becomes completely independent of the fact that we are studying many-to-many traffic once the grooming problem has been solved. In addition to this, the RWA problem has been extensively studied in the literature and it has been proven to be NP-complete. Therefore, we use one of the best existing heuristics for the RWA problem (the LFAP heuristic [18]) which has been shown to use a number of wavelengths that is close to that of a derived lower bound. For a detail description of the LFAP heuristic, the reader is referred to [18].

\section{NumericAl Results}

In this section, we conduct extensive experiments to evaluate the performance of Algorithms 1 and 2. First, we show that the two algorithms use a number of lightpaths that is significantly close to that of the derived lower bound $L$. Second, we compare the performance of the two algorithms on the costs mentioned in the paper including the number of lightpaths and the number of wavelengths used.

We consider two sample networks in our experiments. One is the NJ-LATA network (shown in Fig. 3.(a)) consisting of 11 nodes and 23 bidirectional links and the USNET (shown in Fig. 3.(b)) consisting of 24 nodes and 43 bidirectional links. We randomly generate $K$ many-to-many session requests as follows. The size of a session is randomly selected between $\left[N_{\min }, N\right]$, while members in a session are randomly selected between $[0, N-1]$. Traffic demand of members in a session is randomly selected between $[1,8]$. We study the performance of each algorithm by varying one of the parameters $K, g$ and $N_{\min }$ at a time. Figs. 4.(a) and 4.(d) plot the number of lightpaths $P$ versus the number of sessions $K$ on NJ-LATA 


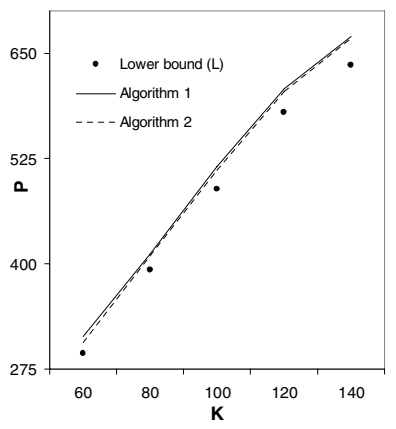

(a)

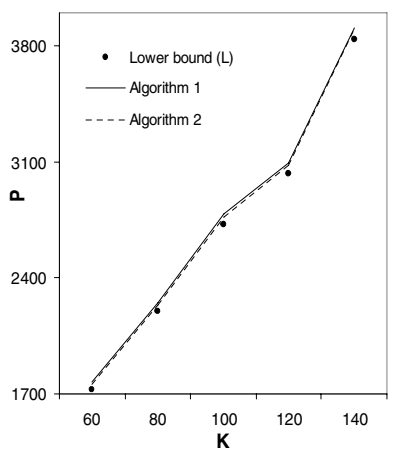

(d)

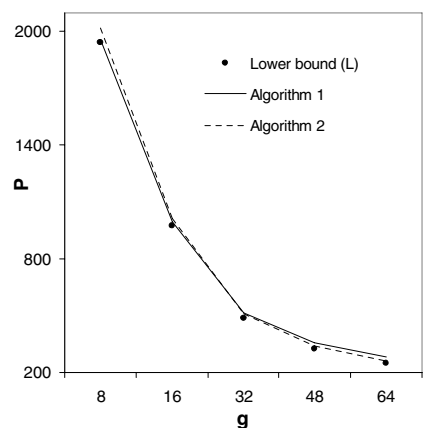

(b)

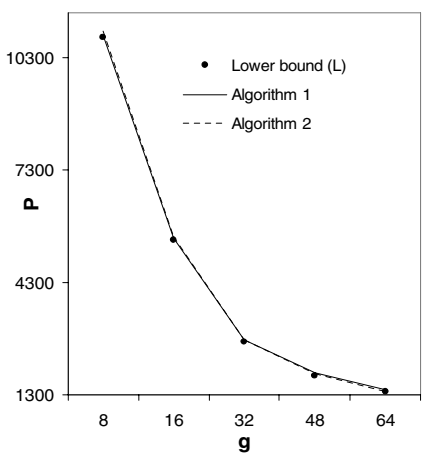

(e)

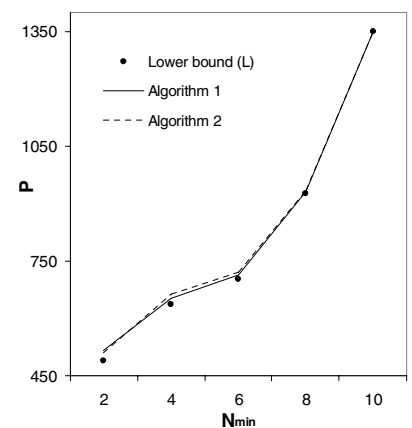

(c)

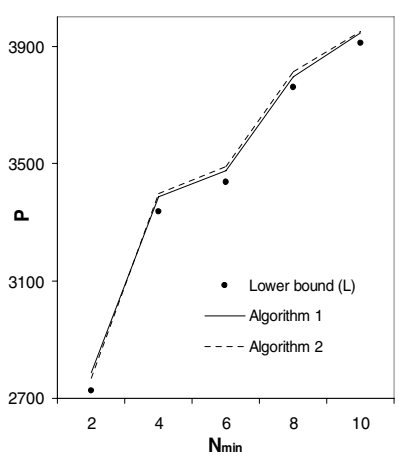

(f)

Fig. 4. Number of lightpaths $P$ versus number of sessions $K$, grooming factor $g$ and minimum session size $N_{m i n}$ on the NJ-LATA topology (a)-(c) and on the USNET topology (d)-(f).

and USNET, respectively with $g=32$ and $N_{\min }=2$. Figs. 4.(b) and 4.(e) plot the number of lightpaths $P$ versus the grooming factor $g$ on NJ-LATA and USNET, respectively with $K=100$ and $N_{\min }=2$. Finally, Figs. 4.(c) and 4.(f) plot the number of lightpaths $P$ versus the minimum session size $N_{\min }$ on NJ-LATA and USNET, respectively with $K=100$ and $g=32$.

We can see from the results in Fig. 4 that solutions obtained from Algorithms 1 and 2 are significantly close to the derived lower bound $L$ on a wide range of network parameters $K, g$ and $N_{\min }$. Since the optimal solution lies between the lower bound and the best of Algorithms 1 and 2, we conclude that the two algorithms give near-optimal solutions and that the lower bound $L$ is tight.

Next, we compare Algorithms 1 and 2 in terms of the number of lightpaths required. Note that the approximation ratio $1+\frac{g}{\left(N_{\min }-1\right) t_{\min }}$ of Algorithm 1 becomes better than the 2approximation ratio of Algorithm 2 when $\left(N_{\min }-1\right) t_{\text {min }}>g$, while it is worst when $\left(N_{\min }-1\right) t_{\min }<g$. Hence, the comparison between the two algorithms is dependent on traffic granularities and on the size of many-to-many sessions.

First, we assume that the size of many-to-many sessions is randomly selected between $[2, N]$ and we compare the two algorithms by varying traffic granularities of sessions in the network. To make the comparison, we assume a static uniform traffic with all sessions in an experiment having the same traffic demand $t$ (e.g., $t_{s_{1}}=t_{s_{2}}=\ldots=t_{s_{K}}=t$ ), where $1 \leq t \leq g$. We generate 50 experiments on the
USNET each with 100 many-to-many session requests as follows. The size of a session is randomly selected between $[2,24]$, while members in a session are randomly selected between $[0,23]$. Given the uniform traffic assumption, each of the 50 experiments is conducted for each value of $t=$ $\{1,4,8,12,16,20,24,28,32,36,40,44,48,52,56,60,64\}$ $(g=64)$ by each Algorithm. We define the normalized number of lightpaths as the ratio of the number of lightpaths $P$ to the lower bound $L(P / L)$ in an experiment. We also define $\overline{P / L}$ to be the average value of all $P / L$ values obtained from the 50 experiments at a particular value of $t$ by a certain algorithm. The corresponding values of $\overline{P / L}$ are shown in Fig. 5.(a).

Second, we assume that traffic demands of sessions are randomly selected between [1,12] $(g=64)$ and we compare the two algorithms by varying the minimum session size $N_{\min }$. At each value of $N_{\min }=$ $\{2,4,6,8,10,12,14,16,18,20,22,24\}$, we conduct 50 experiments on the USNET each with 100 many-to-many session requests as follows. The size of a session is randomly selected between $\left[N_{\min }, 24\right]$, while members in a session are randomly selected between $[0,23]$. Traffic demand of members in a session is randomly selected between $[1,12](g=64)$. The resulting values of $\overline{P / L}$, which is now defined as the average value of all $P / L$ values obtained from the 50 experiments at a particular value of $N_{\min }$ by a certain algorithm, are shown in Fig. 5.(b).

After determining the set of lightpaths for each experiment 

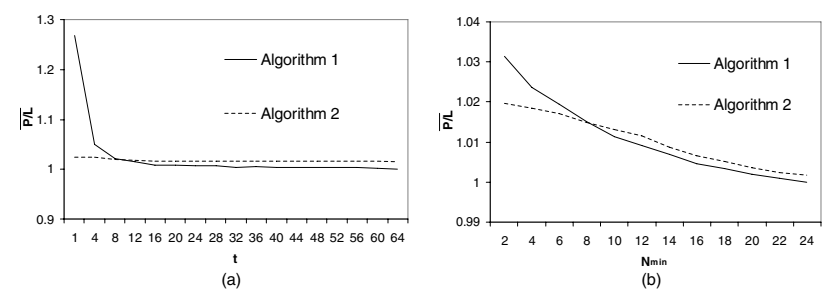

Fig. 5. (a): $\overline{P / L}$ versus $t$ on USNET. (b): $\overline{P / L}$ versus $N_{\text {min }}$ on USNET
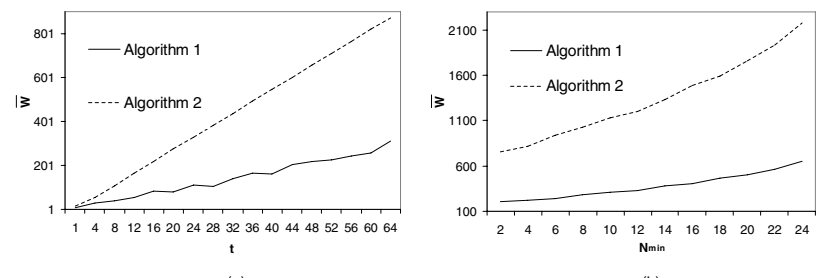

(a)

(b)

Fig. 6. (a): $\bar{W}$ versus $t$ on USNET. (b): $\bar{W}$ versus $N_{\min }$ on USNET

at each value of $t$ (or $N_{\min }$ ) by each algorithm, these lightpaths are routed and assigned a wavelength according to the LFAP heuristic [18]. We define $\bar{W}$ to be the average value of all $W$ values obtained from the 50 experiments at a particular value of $t$ (or $N_{\min }$ ) by a certain Algorithm. The resulting values of $\bar{W}$ versus $t$ and versus $N_{\min }$ are shown in Figs. 6.(a) and 6.(b), respectively.

From Figs. 5-6, we draw the following conclusions:

- Algorithm 2 is more cost-effective than Algorithm 1 in packing and grooming low granularity traffic (e.g., $t \leq$ $\frac{g}{8}$ ), while Algorithm 1 is more cost-effective when traffic granularities of sessions are relatively high (e.g., $t>\frac{g}{8}$ ).

- Algorithm 2 is more cost-effective than Algorithm 1 when the minimum session size is relatively low (e.g., $N_{\min } \leq$ $\frac{N}{3}$ ), while Algorithm 1 is more cost-effective when the minimum session size is relatively high (e.g., $N_{\min }>$ $\left.\frac{N}{3}\right)$.

- Algorithm 2 consumes much more wavelengths than Algorithm 1. The reason is that all the lightpaths generated by Algorithm 2 are between a certain pair of nodes (nodes and the hub). This results in a large number of lightpaths routed on the same link (hence, using a large number of wavelengths). Algorithm 1, on the other hand, distributes the number of lightpaths among the different pairs of nodes in the network through the use of lightpath cycles. This balances the number of lightpaths to be routed on the same link resulting in a fewer number of wavelengths used.

\section{CONCLUSIONS}

In this paper, we have studied the many-to-many traffic grooming problem in WDM mesh networks. First, lightpath cycles were introduced as the optimal solution for a number of special cases where the problem is tractable. Afterwards, two novel approximation algorithms were introduced for the general case. Algorithm 1, which is based on lightpath cycles, has an approximation ratio of $\min \left\{g, 1+\frac{g}{\left(N_{\min }-1\right) t_{\min }}, N-\right.$ $\left.N_{\min }+1\right\}$, while Algorithm 2, which is based on a hub node that collects and distributes traffic, has a 2-approximation ratio. Through extensive experiments, we have shown that the two algorithms perform significantly close to the derived lower bound $L$. We have also compared Algorithms 1 and 2 on the several costs mentioned in the paper including the number of lightpaths and the number of wavelengths used.

\section{REFERENCES}

[1] S. Antonakopoulos and L. Zhang,"Approximation Algorithms for Grooming in Optical Network Design," in Proc. IEEE Infocom'09.

[2] B. Chen, G. Rouskas, and R. Dutta, "On Hierarchical Traffic Grooming in WDM Networks," IEEE/ACM Transactions on Networking, vol. 16, no. 5, pp. 1226-1238, Oct. 2008.

[3] A. L. Chiu and E. H. Modiano, "Traffic grooming algorithms for reducing electronic multiplexing costs in WDM ring networks" IEEE J. Lightwave Technol. vol. 18, no. 1, pp. 2-12, Jan. 2000.

[4] H. Zhu, H. Zang, K. Zhu, and B. Mukherjee,"A novel generic graph model for traffic grooming in heterogeneous WDM mesh networks," IEEE/ACM Trans. Networking, vol. 11, no. 2, pp. 285-299, April 2003.

[5] X. Zhang and C. Qiao, "An effective and comprehensive approach for traffic grooming and wavelength assignment in SONET/WDM rings," IEEE/ACM Trans. Networking, vol. 8, no. 5, pp. 608-617, Oct. 2000.

[6] O. Gerstel, R. Ramaswami, and G. Sasaki, "Cost-effective Traffic Grooming in WDM Rings," IEEE/ACM Transactions on Networking, vol. 8, no. 5, pp. 618-630, Oct. 2000

[7] R. Dutta, S. Huang, and G. N. Rouskas, "Traffic grooming in path, star, and tree networks: Complexity, bounds, and algorithms," IEEE J. Sel. Areas Commun. vol. 24, no. 4, pp. 66-82, Apr. 2006.

[8] K. Zhu and B. Mukherjee,"Traffic Grooming in an optical WDM Mesh Network," IEEE Journal on Selected Areas in Communications, vol. 20, no. 1, pp. 122-133, Jan 2002.

[9] J.Q. Hu and B. Leida, "Traffic grooming, routing, and wavelength assignment in optical WDM mesh networks," in Proc. IEEE Infocom'04.

[10] R. Dutta and G. N. Rouskas, "Traffic grooming in WDM networks: past and future," IEEE Network, vol. 16, no. 6, pp. 46-56, Nov./Dec. 2002.

[11] R. Ul-Mustafa and A. E. Kamal,"Design and provisioning of WDM networks with multicast traffic grooming," IEEE J. Select. Areas Commun., vol. 24, no. 4, pp. 37-53, Apr. 2006

[12] G. Chowdhary and C. S. R. Murthy,"Grooming of multicast sessions in wdm mesh networks," in Workshop on Traffic Grooming, 2004.

[13] A. E. Kamal "Algorithms for Multicast Traffic Grooming in WDM Mesh Networks," IEEE Communications pp. 96-105, Nov. 2006.

[14] A. Billah, B. Wang, and A. Awwal, "Multicast traffic grooming in WDM optical mesh networks," in Proc. GLOBECOM'03.

[15] H. Madhyastha et al., "Grooming of multicast sessions in metropolitan WDM ring networks," Computer Networks, vol. 49, pp. 561-579, 2005.

[16] A. Rawat, R. La, S. Marcus, and M. Shayman, "Grooming Multicast Traffic in Unidirectional SONET/WDM Rings," IEEE Journal on Selected Areas in Communication, vol. 25, no. 6, pp. 70-83, August 2007.

[17] M. Saleh and A. Kamal, "Many-to-Many Traffic Grooming in WDM Networks," accepted for publication in the IEEE/OSA Journal of Optical Communications and Networking.

[18] H. Siregar, H. Takagi, and Y. Zhang, "Efficient routing and wavelength assignment in wavelength-routed optical networks," Proc. 7th AsiaPacific Network Operations and Management Symp. Oct. 2003.

[19] M. Saleh and A. Kamal, "Design and Provisioning of WDM Networks for Many-to-Many Traffic Grooming," to appear in proc. IEEE Globecom'09.

[20] R. Ul-Mustafa and A. E. Kamal,"Many-to-one Traffic Grooming with Aggregation in WDM Networks "IEEE Journal on Selected Areas in Communication, vol. 24, no. 8, pp. 68-81, August 2006.

[21] Long L. and A. E. Kamal, "Reducing network cost of many-to-many communication in unidirectional WDM rings with network coding," accepted for publication in the IEEE/OSA Journal of Lightwave Technology.

[22] C. Diot et al."Multipoint Communication: A Survey of Protocols, Functions, and Mechanisms," IEEE J. Select. Areas Commun., vol. 15, pp. 277-290, 1997.

[23] B. Quinn and K. Almeroth, "IP multicast applications: challenges and solutions," IETF Request for Comments (RFC) 3170, Sept. 2001. 